\title{
Snapchat Memory and Youth Digital Sexual Cultures: Mediated temporality, duration, and affect
}

\author{
Sarah Handyside ${ }^{\mathrm{a}}$ and Jessica Ringrose ${ }^{\mathrm{b}} \mathrm{a}$ Centre for the study of Women and

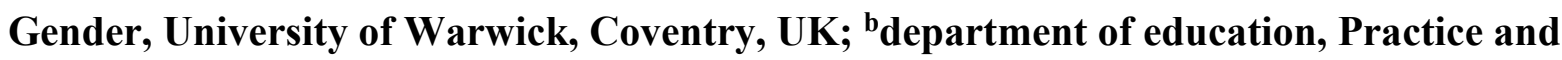 \\ Society, UCL institute of Education, London, UK
}

\section{Sarah Handyside and Jessica Ringrose}

\begin{abstract}
This paper explores how the photo and video-sharing app Snapchat mediates memory and intimacy, using focus group data with 18-year-olds. We use Bergson's ideas about duration and Deleuze and Guattari's theories of affect and assemblages to think about how the digital affordances of 'disappearing' Snapchat technology reshape memory and intimacy in youth sexual and relationship cultures. Our findings illustrate that Snapchat offers a temporal fastness and ephemerality - but also forms of fixity through the screenshotting of 'disappearing' snaps. Because judgement from peers cannot take place publicly within the app, offline discussion of Snapchat activity gains significant traction, making interview accounts of Snapchat use highly relevant. Our analysis of discussions of 'Snapchat memory' explores the gendered aspects of performative 'showing off' and sexual scrutiny, considering what happens when snaps do not disappear and how Snap exchanges can be used as relationship currency; for instance exploring how some participant's challenged Snapchat related slut shaming through their uses of humour. Overall we show how Snapchat is mediating youth intimacy, highlighting the reconditioning that occurs between and across the digital world of Snapchat and the physical world of its youth users - evidence of the blurring of online and offline experiences that disrupts digital dualisms.
\end{abstract}

'Enjoy fast and fun mobile conversation! Snap a photo or video, add a caption, and send it to a friend. They'll view it, laugh, and then the Snap disappears from the screen-unless they take a screenshot!' (Snapchat app description, 2015).

'Say you a freak, show me how freaky

And she got moves like bad gyal RiRi

Said he hit it right, go $H \cdot A \cdot M$ (hard as mother**cker) when he tap that

You last ten seconds, man, you're a Snapchat' (Freak of the Week LYRICS, Krept \& Konan)

'Psychic states [...] unfold in time and constitute duration' (Bergson 1950: 224). 


\section{Stories, snaps and screenshots: what is Snapchat?}

Snapchat is a photo and video messaging app with an unusual temporal structure. Its uniqueness lies in the transience - more specifically, the possible transience - of the images and videos that users share with each other, called 'snaps'. With a lifespan stipulated by the sender of between one and ten seconds, snaps are ostensibly temporary, ephemeral things. The receiver can however choose to screenshot and preserve a snap, imbuing it with a fixity out of the sender's control (the app will inform them that a screenshot has been taken). Snapchat is aware of this potential permanence; screenshotting is laid out as a playful option in the company's marketing text.

Additional Snapchat functions include a 'My Story' option, enabling users to publish snaps to their entire friend list for 24 hours, creating an updatable (though still transient) narrative personal profile more akin to a social network site (SNS) than a traditional messaging platform. Meanwhile, individual users' activity levels are translated into a score that increases with each snap sent. This number is displayed next to that person's username, publically showcasing their levels of Snapchat use. Snapchat friends of this user can then deduce when they are exchanging snaps with somebody else, by monitoring whether their activity score is increasing.

Many users and indeed academics enthuse about the carefree enjoyment Snapchat can produce, often focusing on its quirky temporality (Jurgenson 2013, 2014; Velez 2014). Yet this temporality has courted controversy. Seen in a range of international news headlines such as 'Snapchat: Sexting tool, or the next Instagram?' (CNN, January 2013) and 'Will Snapchat's new update stop people from sexting using the app?' (Telegraph, September 2015), Snapchat gained early notoriety as the so-called app built for 'sexting', its quick-to-vanish imagery being seen as the ideal medium for transmitting explicit content (see, for example, Poltash 2013; Roesner et al 2014; Utz et al 2015). Returning to the 'Freak of the week' song lyrics above, Snapchat has become embedded in a sexual lexicon adapted to account for time, documentation and memory. In the lyrics, Snapchat refers to both the fastness of Snapchat - the short time period for which the protagonist can 'hit it right go HAM' - and presumably his desire to (temporarily) capture himself to remember doing so. Meanwhile, numerous websites offer public, downloadable lists of 'female Snapchat usernames who enjoy Snapchat sexting'. Responding to this public construction of Snapchat's sexualised technological affordances, in

\footnotetext{
${ }^{1}$ Snapchat Sexters, <http://snapchatsexters.com/> Accessed 22 July 2015.
} 
2014 Eton College banned the app in an effort to curb sexual snapping (Business Insider, January 2014).

Thus Snapchat's distinct temporality and user affordances have unfolded in ways viewed as having specific impacts, risks and possibilities presented by digital culture around gender and sexuality but also age. In this paper we explore how teenagers use Snapchat and how its temporality and ephemerality shape those teenagers' sexual cultures; that is, their networked subjectivities, connections, intimacies and relationalities online and offline. Our discussion seeks to contribute to research on social media networks that is exploring the discursive, affective and material aspects of how the technological affordances mediate relationships, gender and sexuality in new ways (Chambers, 2013; Van Doorn 2011). We specifically wish to contribute some analysis around temporality, duration and memory, drawing on the work of Henri Bergson and Gilles Deleuze to rethink Snapchat mediations of gender and sexual relationality. We focus on how the supposed disappearance of the snap (image-object) reshapes memory, and explore new durations of jealousy and possessiveness, although we also attend to how Snapchat's ephemerality leads to playfulness and learning around gender and sexual relationship norms. As such, we examine some of the intimacies of youth sexual encounters and romantic relationships as mediated through Snapchat's uniquely part-ephemeral, part-permanent lens, questioning how this particular application's unusual temporality relates to youth digital sexual cultures.

\section{Background: New Media, Snapchat research, ephemeral entanglements and intimacies}

'New media' encompasses a wide range of digital platforms that offer new ways to 'produse' (Bruns, 2011) connect and network. New media and social media research explores advances in mobile technology and near-universal Western internet access. danah boyd and Nicole Ellison, for example, define an SNS as a 'web-based service' through which users can create a personal profile, collate a list of friends, and view both their own connections and those made by their friends (boyd and Ellison 2008: 211). Snapchat both meets and disrupts these criteria. Its unusual temporality contrasts with the archive functions of, for example, Facebook and Twitter, in which content remains retrievable. We wish to explore this non-retrievable and nonreturnable dimension to Snapchat as creating new temporal and subjective relations to the images (objects) that disappear.

Ephemerality is unsurprisingly a key theme of studies that focus on Snapchat. Utz et al's (2015) study of jealousy and Snapchat affirms the popular opinion that Snapchat's ephemerality, as well as its privacy, are key drivers for flirtatious or sexual communications. 
Similarly, Koefed and Larson (2016: 2) in their comparison of young people's photo sharing on Snapchat and Instagram note that Snapchat "lacks the persistent content [of Instagram] (eg a feed with archived photos and videos)". Indeed the Guardian article 'Facebook is over" written by Lucia Hagan 16, notes "There are only two types of social media anyone my age uses: Snapchat and Instagram. Snapchat is for giving everyone a constant insight into your life, without it being as annoying as posting loads of videos and photos on to Instagram (Snapchat posts disappear). Instagram is basically the same thing, except your uploads are more spaced out. The only people I know who use Facebook are my parents; mostly it's a place where people dump their non-Instagram-worthy pictures every couple of months."

Thus, intimacies are mediated in and through the unique temporality and duration of Snapchat (constant and disappearing) enabled through its technological affordances. Experiences of the temporality of the app have been theorised as 'affective' that is inducing of specific embodied reactions (Massumi, 2001). For instance, Velez (2014) describes the app as engendering 'ephemeral bursts of affect', through quick exchanges of 'excitement, curiosity, or boredom' that can feel like a 'secret' because of their transience. Meanwhile, Jurgenson (2013) suggests that Snapchat breaks the boundary between lived experience and documentation of experience constantly rupturing and blurring this boundary.

This research is valuable both as highlighting Snapchat's unique temporality, and in their dismantling of the outdated notion of 'digital dualism'. Now, 'the digital and physical are increasingly meshed' (Jurgenson 2011); this is a strong trend in research on digital mediation as a process that dissolves the dualism between real-life and online space (see, for example, Kember and Zylinska 2012). This dissolution is apparent throughout our findings in terms of how youth friendships and relationships and gender and sexual cultures operate through the app; Snapchatting is therefore an active part of how relationships and intimacies are now formed, not merely a means of documenting them in virtual space.

Perceptions of Snapchat users' offline lives are also manipulated digitally via the app; 'it's easy to make people think you're having a much cooler life than you are', admitted one focus group participant, as we explore. When asked whether not having Snapchat was an option, another participant stated 'I think it's too far gone now not to have it anymore.' She illustrates the thorough, if messy entanglement of Snapchat and offline life - precisely what Sarah Kember and Joanna Zylinska (2012) have identified in their call for thinking about new media not as a series of objects but 'in terms of the interlocked and dynamic processes of mediation' (Kember and Zylinska 2012: 1). 
In our reading, Velez and Jurgenson ignore the tension that Snapchat generates between ephemerality and permanence, experience and documentation and how this is reshaping intimacies. Indeed, they do not engage with wide-ranging concerns over the sexualised risks or harms associated with youth users of Snapchat, visible in the mass public hysteria surrounding discussions of youth sexting and particularly the exchange of 'nude' images. Snapchat has been positioned by mainstream mass media as the perfect sexting platform because of the assumed disappearance of snaps and the simultaneous ability of the app to capture the image, therefore extending its duration and potential stigmatisation (Trottier, 2013). This fear is explicitly highlighted in Charteris et al's (2016: 3) research on parents views of Snapchat, which argues 'disappearing' media like Snapchat is part of teen 'underlife', which creates new anxieties around regulating youth (hetero)sexuality. In their study parents respond to Snapchat ephemerality and (in)visibilities with desires to find, see, and ultimately control 'risky' sexualised content on social media as part of adult desires for childhood innocence. Our study explores similar content but from the perspective of the young Snapchat users themselves. Rather than solely focusing on the economies of visibility, regulation and containment of youth use of ephemeral media like Snapchat, encapsulated in the anxieties over youth sexting, ${ }^{2}$ we want to think through how Snapchat remediates the very matter of youth sexual cultures and intimacies. Through a discussion of memory, liveliness and duration, we explicitly trouble the idea that Snapchat is simply a form of 'disappearing' social media.

\section{Mediated memory: Duration and affect in social networks}

Henri Bergson's statement that 'no two moments are identical in a conscious being' (Bergson 2007: 137) reminds us that even as moments from the past are recalled, they are changed, reconditioned by events and consciousness that have unfolded since. Memory is a productive force, ensuring that the past is never experienced as it was but as difference. Crucially this difference is both individual and subjective, experienced uniquely by individuals transitioning through time. Such productive altering and reshaping of the past by the continually unfolding present forms the core of Bergson's duration theory, an understanding of time experience that is individual, subjective and intuitive, continually psychically shifting and changing (Bergson 1950: 224). Memory thus mediates possible subjectivities and

\footnotetext{
${ }^{2}$ The legal implications of image exchange and youth sexting in varying international contexts have been reviewed extensively elsewhere (see for instance Karaian, 2013 and Hassinoff, 2014). Circulation of sexually explicit and nude Images of children under 16 years of age is illegal in the UK. Our focus in this paper is not upon the legal implications of sexually explicit Snapchat images but more upon how Snapchat digital image exchange is reshaping youth sexual cultures more broadly.
} 
relationalities; it is 'the present's mode of access to the past. The past is preserved in time and the memory-image [...] can be selected according to present interests' (Grosz 2004: 178). Digital media images and social media processes like posting, tagging and sending of course interfere into this memory-image selection process in significant ways.

A Bergsonian analysis of Snapchat in particular, is useful in taking account of how the application mediates the real and the virtual of time through posts and images. For instance, according to Goodings and Tucker (forthcoming), Bergson is useful in asking questions of 'how people come to produce and know themselves in and through social media?' 'What kinds of challenges and dilemmas are faced when living through social media?' Goodings and Tucker (forthcoming) explore Facebook timelines through a Bergsonian frame, suggesting that the stuck and rigid temporal artefacts of the timeline image memories on Facebook interfere with a Bergsonian sense of expansion and shifting of memory, creating problematic structures for users to navigate. Snapchat's ephemeral temporal structure is very different; snapping is fast, and snaps typically disappear quickly, yet have continuing effects without a physical manifestation. Snapchat is a social media app that therefore alters relationality through networked events, and mediates memory in ways that have not yet been mapped and demonstrated.

In our analysis, Snapchat both illustrates and disrupts duration's continual reconditioning of the past. It sometimes retains visual reminders of what has happened, in screenshots and activity scores, and yet also ensures that images disappear or dissolve, left only to be discussed and therefore embellished and edited without physical reference points. Snapchat is, as we demonstrate, frequently discussed by young people after particular activity has taken place, but these discussions alter both what originally happened, and as the emotional responses of those subjects. As such, we argue that Snapchat as a mediating technology actually transforms and interferes into another (incomplete) metaphorical representation of duration. Snapchat's images are intra-acted with (here we draw on a Baradian relational notion of posthuman intra-action with social media posts having messy, complex lines of interference) past the point of viewing, which we need to consider carefully (Warfield, 2016). As Grusin (2010) has suggested in his discussion of mass media "premediation" where perpetual news crisis affectively shape what can be known about the world, Snaps interference with memory also impacting possible futures. Thus, snapchatting enables 'specific durations' with its own measures, spans and fluxes and manifold dynamical unfolding effects and affects (Grosz 2011).

Bergson's influence is strongly apparent in the work of Deleuze, in his interest in transformation and becoming, and directly in such statements as 'the virtual insofar as it is 
actualized, in the course of being actualized, it is inseparable from the movement of its actualization' (Deleuze 1988: 42-43). Likewise, Deleuze and Guattari centralise difference, change and transition in their theories of how desire and capacities for bodily affect 'flows through and between (human and nonhuman) machines/assemblages/bodies in complex ways'. Media scholars have suggested affect is particularly important for understanding how social media posts actually have a variable relational force of connectivity amongst users as noted in Paasonen, Hillis and Petit's (2015) work on 'networked affect' generated through connective online spaces like Facebook. Assemblage theory has been applied to explain networked relationality enabled through social media accounts such as public affect generated through Twitter feeds (Papacharissi 2014). The ways networked friends create new 'affective assemblages' and economies of visibility and looking via semi-private sites like MySpace, Bebo, Facebook or BBM criss-cross and reshape material friendship cultures has also been explored (Ringrose and Coleman, 2013; Van Doorn, 2011). SNSs create new digital dimensions of school-based friend groups, what we might call affective mediated web-like structures through which various emotions circulate and interact, with variable "intensities" shaping what users/bodies can or can't do (Ringrose, 2011; Ringrose and Harvey, 2017). These structures disrupt traditional notions of public and private and online and offline and individual and group (Donath and boyd 2004; boyd and Heer 2006; Marwick and boyd 2014). For instance, they force privacy to be negotiated in sometimes highly visible ways, and online public display to be part of the formation of offline friendships and relationships.

We use Deleuzo-Guattarian ideas of social media affects and assemblages to foreground the complex and shifting dynamics of power and desire at work in social media school-based friend groups. We bring this together with Bergson's attention to duration (in which the past is never static, and memory is individual, subjective, and productive) to think further about social media affects - particularly to explore how young people's relationship cultures (friendship, intimacy and sexuality) are shaped by group use of Snapchat. Specifically, we explore how the complex and productive temporality of Snapchat mediates how gender and sexuality are performed and negotiated and remembered. We demonstrate durational fluxes and how a range of subjective states are generated and managed in relation to snap content.

\section{The Research}

Our methodology sought to explore Snapchat dynamics through interviewing. We ran two semi-structured focus groups with 18-year-old mixed-gender students at a large state school in north east England; six in the first group (two boys and four girls) and two in the 
second (both girls). One participant was British Asian, the others all white British (the school's student body is almost entire white British). Participants' sexualities were not disclosed.

An open discussion format was deliberately chosen, with the interviewer asking general questions about the application and the participants shaping their own debate in ways that were designed to elicit stories. Participants were, for example, asked to describe their strongest memories of Snapchat, and particular psychic states that they had experienced in relation to the app. Such individual storytelling helped shed light on subjective experiences of duration through Snapchat, the contrasting psychic states at play as power dynamics shift, and the most personally significant intimacies experienced within and through the app. Participants were not asked to explicitly compare Snapchat to other forms of social media, and this would potentially be a fruitful area for future research.

Participants voluntarily joined the groups following publicity in assemblies; while they all knew each other, they were not all necessarily close friends. The ethical dimensions of the interviews were discussed with the participants, who agreed to ground rules around keeping any sensitive aspects of the discussion within the groups. An interview methodology is particularly important for Snapchat as it allows for an analysis of the talk generated about content that is no longer available to view. Thus, whereas with Facebook or Twitter a digital artefact may remain, with snaps we are dealing with memories and need to rethink the relationship between time, image and meaning (Coleman 2014).

Snapchat's ephemerality and the disappearance of content means that the snaps being used to shape particular judgements have frequently vanished by the time that such assemblage is taking place, leaving more space for speculation. We explore this below by considering the role of gossip and rumour-spreading after particular Snapchat events, as well considering how participants use strategies like humour to 'reshape' upsetting Snapchat episodes.

\section{'It is a very like, showing off kind of thing': Impressions and perceptions, ambiguity and jealousy}

Two contrasting temporal structures operate within Snapchat; fast, fleeting, disappearing snaps sent with lifespans of a few seconds, and 'My Story', in which users post snaps for friends to view an unlimited number of times within 24 hours. These can be images or videos of up to ten seconds, and may include textual captions. 'Story' evokes both narrative temporal structure and individual creativity; both elements invite an examination through the lens of duration. 
Richard underlined that a positive facet of 'My Story' is that feedback on snaps must take place privately, through a message, which makes Snapchat potentially more attractive than other social media:

RICHARD: It's kind of like, you know how you can put things on your story and people can't like or favourite so you don't know if people are judging you or not? [laughter, agreement] So, like, you can't tell if people - cos if you put something really stupid no one can be like 'yeah that's terrible', cos, if like on Twitter if you get like no likes or favourites it's like 'oh, great, no one cares about my tweets', while Snapchat, you can't really get that judgement from peers.

Much Snapchat judgement takes place offline, or through private snap exchanges following a public broadcast. Because of this, and because the original posts don't last in time, psychic projection plays a prominent role on the app, with users predicting how they think others will respond to snaps. In other words, imagined judgement gains significant strength on Snapchat. This is a facet of productive memory; with no physical signifier of particular judgements, second-guessing becomes powerfully influential. And second-guessing creates ambiguity and multiplicity. Indeed, later Richard contradicted his statement, demonstrating that that Snapchat is by no means a judgement-free zone and exerting a particularly gendered assessment of Snapchat behaviour:

RICHARD: In terms of like, showing off on the stories, it's usually all girls. I see like 'out tonight with the lasses'. No one cares. Like, I never see a guy Snapchat stories saying...

Much of the rest of the group immediately cut in with shouts of agreement. Here, 'showing off' is discussed disparagingly as a typically female Snapchat behaviour (by a mixed gender group). A 'constitutive process' (van Doorn 2010: 586) by which technology and gender mutually form each other is apparent. Showing off on Snapchat classifies a user as a girl, while equally, being a female user of Snapchat means that one's stories are more likely to be trivialised as showing off.

Yet 'My Story' is a tool specifically intended for public broadcast within a group. How, then, does such broadcasting shift to being characterised disparagingly as showing off? Frequency of updates might be one element, and despite Richard's claim of banality and 'no 
one cares', Snapchat performances are intimately tied affective flows of desire. Richard himself demonstrated this, explaining how stories can be carefully curated to form idealised representations of users' lives:

RICHARD: It is a very like, showing off kind of thing, like, you can post something of yourself doing - you can - it's easy to make people think you're having a much cooler life than you are, cos if you're like, you can take a blurry Snapchat of the floor and be like, 'oh, I'm so mortal', and really you're just...

'Mortal' here is regional slang for drunkenness, usually associated with parties or nights out, hence this kind of story's association with a 'cool' life. Richard links showing off with the curation of a finely edited version of one's life, through which truth is manipulated to present a specific impression through the temporal impermanence of snaps. Snapchat's transience means that a non-screenshotted story vanishes within hours, kept alive only in discussion. Since fewer signifiers or visual artefacts of events are left behind, Snapchat lends itself more to an affective memory of the event, rather than a reviewing of an actual image, as may be apparent on Facebook, for instance. A Bergsonian model of memory, which helps us to understand Snapchat producing difference and multiplicity, is clearly apparent.

Jealousy has been identified as particularly rife on Snapchat (Utz et al 2015); not necessarily an individualised jealousy but rather a transpersonal force due to the app's ability to rank relationships. We propose that the particular fluxes and flows of this emotion can be compared to a specific representation of duration drawn by Bergson - namely, sympathy. He suggests that sympathy incorporates both an indivisible progress of interlinked parts (from repugnance, to fear, to sympathy, to humility), and the process of imagining oneself in the future, in the position of another (Bergson 1950: 19). Jealousy works in a similar way, involving both the process of imagining oneself in another (better) position, and heterogeneous but connected elements including admiration, a sense of lack, and desire. Such heterogeneous but indivisible flows of affect through time are frequently at play within Snapchat.

Richard shows how Snapchat is deliberately used to engender jealousy and to create so-called 'cool' subjectivities. Although all social media offers the opportunity to do this, Snapchat specifically encourages jealousy of scenarios continuously re-imagined as an aftereffect of a snapped but disappearing image. Ambiguity and creativity saturate the app, ensuring that power relations are particularly unstable. 
Jealousy thus relates to an individual's time experience; it involves both a past/present characterised by lacking something, and an imagined future in which that gap is filled. It also connects to an individual's group networks. Within Snapchat, it is perhaps precisely because those elements of time experience and group networking are so complex and shifting that jealousy is such a powerful and frequently occurring emotion.

By tying together these threads of networked emotion, ambiguity and jealousy, an initial answer to the overarching question of what sexual subjectivities memory produces can be sought. Hannah was asked to describe the kind of Snapchat activity that would be most likely to be talked about later:

HANNAH: I saw...right ok, this is bit stupid, but I - this boy was supposed to texting this girl. Or it seemed that way anyway. And then he put a picture on Snapchat of him and a different girl. But that different girl's face was like blocked, it was like, you couldn't see her face cos something was like in the way of it. So - and then - I dunno - I started - I remember, I like made a point to talk to my friends about it. Cos it was like, an odd thing, and I think people - yeah like that was I think that was done like cleverly, because, you couldn't see who the girl was but you could tell it wasn't the girl it should have been.

Here, ambiguity is both acknowledged by Hannah as a common factor in negotiations of romantic relationships ('it seemed that way'), and perceived by her as a deliberate, 'clever' strategy taken by the boy to position himself with multiple girls. Such positioning relates to established notions of masculinity performed on social media through sexual popularity (Harvey et al., 2015), so the presentation of a normalised gender identity is apparent here. Yet the ambiguity of the image provokes an extra layer of intrigue, ensuring that the boy's potential flirtations or sexual relationships become the topic of offline and online discussion. Jealousy is not explicitly present - certainly Hannah does not suggest she or anyone else felt it - but her closing reference to 'the girl it should have been' is telling. Here, Hannah both points towards a set of unwritten rules governing the negotiation and display of intimacy on Snapchat, and indicates that an ambiguous image could be used to provoke jealousy from someone who 'should' possess something.

Snapchat is the ideal medium for this kind of ambiguity because not only can the visual format of snaps, as shown here, be manipulated to hint at multiple meanings, but the snap will also vanish after a certain amount of time and will therefore be distanced from the original 
poster, even if it is screenshotted somewhere else. Snapchat becomes a tool with which to suggest what sexual subjectivities and intimacies might be.

The 'truth' on Snapchat then, as everywhere, is a slippery thing. Memory, both within and of Snapchat, produces multiple and sometimes contradictory interpretations of events. This is partly due to Snapchat's ephemerality, which encourages slippages between remembering and physically re-seeing an image. It is also partly due to subjective attempts to recondition past events in a flattering light, like Hannah searching for the right words to ensure she is not perceived as a gossip. These multiple interpretations lead to jealousy, which in itself inhabits an unusual temporality, potentially exacerbated by Snapchat. If a snap has vanished, it is impossible to prove what originally happened, and easier to reshape in the future. Jealousy, after all, does not have to be of what someone actually has, just what they appear to have. These slippages also inform harsh and gendered judgements of particular Snapchat behaviours, as we shall show.

\section{'It's always the girl that gets the stick for it': Snapping sexual double standards}

Richard's claim above that girls' are particularly guilty of 'showing off' on Snapchat (and other social media) was hotly debated:

CLARE: What you're wearing-like, on your-if you take a selfie, and like maybe you've got, like, a bit of cleavage, then a boy will be like 'oh, she wants some', and send, like just send you like... [agreement from other girls] Like, you know, it's just, they, they take things the wrong way, like, maybe you just, that's how you dress, and, like, you know, you're going out.

Alice provided a similar example:

ALICE: $\quad$ When I had long hair, like, and like, it was when I'd put it up in a bun and it would go really really crazy and cool once I took it out, I would put that on Snapchat because - like, on my story, because I dunno - I thought my hair was really cool and I always used to get - there's this one guy who continually was like - he'd send me Snapchats and he was like 'sex hair', and I was like 'no! [laughter] No it's not! I'm going to bed!' 
Snapchat's digital landscape here entangles particularly messily with offline sexual relationships and intimacies; the inference is that the girl 'wants' not just explicit snaps, but physical sexual contact. Selfies operate as both objects and practices, or gestures, 'created, displayed, distributed, tracked and monetised through an assemblage of nonhuman agents' (Senft and Baym 2015: 1589). Clare and Alice here demonstrate such assemblage, showing how an image intended as an innocuous record of an evening out or a new hairstyle can easily develop an alternative narrative, with (male) audiences reconfiguring its meaning around reading girls' embodiment as sexualised. Comedy operates here as a tool for reframing past episodes; Clare and Alice both sought laughing agreement from the other girls. Memories of Snapchat activity both hang on visual 'anchors' (the selfies the girls refer to), and are remediated, reformed through humour. Clare and Alice here seemed able to take back some agency over the harsh sexual judgements they had been subject to, going some way to trouble the simplistic sexualised readings boys had applied to their selfies.

The notion of girls' everyday snaps being sexualised by peers in ways that sometimes feel funny and sometimes out of their control was returned to in the follow-up interview, in which the group explicitly discussed the sexual double standards whereby girls' snaps were taken too seriously and out of context:

HANNAH: I think girls are judged more negatively I guess. Surrounding things like the hair thing and stuff but then I-I dunno. Cos then I guess if a boy's - if a boy's doing that and making it all obvious people are like - people just kind of laugh at it and think 'oh that's funny', but if a girl does it, you kind of think 'oh, she's a bit of a slut.'

RUTH: $\quad$ Yeah! That happens all the time. It's so annoying.

HANNAH: Yeah, like, people won't see it as funny, whereas if it was a boy people would probably laugh and see it funny.

INTERVIEWER: If a boy posted a photo of messy hair?

HANNAH: If a boy did it, yeah. Whereas a girl, is probably gonna be a lot more negatively judged about that. 
INTERVIEWER: And do you have any idea why that is?

RUTH: [Sarcastic] Cos girls aren't allowed to have fun. That would be awful.

HANNAH: Yeah, there is always a double standard isn't there?

RUTH: $\quad$ Yeah.

HANNAH: With everything.

RUTH: It's like, it's like when me and this guy were - like doing stuff on Snapchatum, he was like, people found out, and everyone - they kind of took the piss out of him in, like a funny way, but everyone, or apparently everyone was talking about how much of a slut I was, when it was like, it's not a one person thing, it's like a two people thing.

Hannah and Ruth here cut to the heart of the double standards of gendered judgement that shape their lives on and off Snapchat - standards in which Hannah at least is knowledgeable complicit, as the term 'you kind of think' underlines: "such sexual double standards are hardly new, [but] technology provides new ways for value to circulate through images, and for value to become materially marked on particular bodies as part of that process" (Ringrose et al., 2013: 13). Examining the temporalities at play here, it seems that sexualised snap exchange places a far heavier burden on girls than boys. Through a lens of Bergsonian duration, the event of Ruth sharing sexualised snaps with a boy imbues her with the label 'slut', colouring her past with a sticky and undesirable identity. The boy, by contrast, apparently experienced a far more transient and less weighty 'taking the piss'.

Private snap exchanges are interesting here in that they are invoked through gossip. The vague 'doing stuff' that Ruth refers to - the intimate exchange of explicit snaps with her boyfriend - is subsequently discussed by her online and offline networks, without them holding fixed visual reference points. It is precisely the lack of such physical evidence that allows the virtual reality of Snapchat to produce such complex and variable sexual subjectivities, because memory tends to focus more on what a user has heard happened than what they have physical evidence for actually happening. Significantly, snaps are read through a sexual double standard relating to humour for boys and judgement for girls: Ruth's boyfriend is treated comically and 
Ruth is endowed with the identity 'slut'. Such gossip constitutes another kind of creative Snapchat storytelling - and has a temporal facet.

Comedy is exposed as gendered here. A boy who posted a photo with messy hair like Alice's, would apparently be judged as funny, whereas 'slut' carries weighty connotations of previous, sexualised activity - Ruth is assumed to have behaved in certain ways, done certain things. 'Funny' keeps the incident in the present, endowing the boy with nothing more than a sense of humour in that moment. Girls seem to be more vulnerable to being assigned sexual subjectivities that colour their entire pasts.

Ruth continued to describe some of the double standards attached to sexual image exchange on Snapchat:

RUTH: $\quad$ Whenever like people do things like send nudes on Snapchat, it's always the girl that gets the stick for it. Like, there's literally two people, but then everyone'll like, they'll come up to whoever did it, and they'll be like, 'everyone's calling you a slut', and it's like 'well, you know, I don't do it on my own...'

HANNAH: [Sceptical] Mmm.

RICHARD: The thing is, cos guys ask for it more, you never get a girl going 'ooh, give me dick pics' kind of thing. [agreement] It's not, that's just not how the girls work, I don't think personally but I think girls are probably more willing to give, I don't know, I suppose they're more...

'I don't do it on my own' renders Ruth's statement highly personal and understands the recipient of images as being as complicit as the sender - image exchange as a balanced activity. By contrast, the other participants (who potentially did not have as direct, personal and evocative experience to draw on), struggled to find a balance of agency between willingness and refusal, active requests and subtle hints. Statements seemed to characterise boys as passive image collectors, building up valuable troves, and girls as active image creators and transmitters, but this contrasts with the active demands of boys who, in Richard's words, 'ask for it more'. It seems that being willing to send sexual imagery is a more harshly judged Snapchat activity than directly asking for such imagery.

The double standards exposed through Snapchat activity are gendered, then, in ways that link with Bergsonian and Deleuzian temporalities. Girls' identities are far more at risk than 
boys' of being dramatically altered by labelling and gossip that colours and disrupts whatever identities they presented previously. Their Bergsonian 'unfolding' of 'psychic states' through Snapchat seems fragile, disruptable by a single action.

Snap exchange, in other words, is not an evenly balanced activity. There is a temporal contrast here too, where if boys are collectors keeping records of the past, girls are transmitters, acting fleetingly in the present. Yet this contrasts again with girls being more vulnerable to sticky identity judgements that colour their pasts.

Clare then attempted to explain this imbalance:

CLARE: It's easier - probably - ah, I know this sounds really bad, but it's probably easier for the girl to send it, like...

HANNAH: Yeah. I think the problem is less - well it is still but a bit of a less of a stigma for girls to as well.

RICHARD: Mmhmm.

HANNAH: A little bit. I do think there is obviously a lot but I think it's slightly more acceptable because people always go on about it don't they?

The notion of it being 'easier' for girls to send sexual images of themselves than boys is particularly illuminating. There is 'less of a stigma' for girls to sexualise themselves visually precisely because, as we have explored, images of girls automatically carry sexual currency in Hannah's words, 'people always go on about it'. We can clearly see here the sexual double standard around girls, positioned as both victims and drivers of moral judgements in image transmission (Ringrose et al, 2013).

Memories of Snapchat incidents form perceptions and consequent judgements of users that can be hard to shake, particularly for girls. These judgements are formed both through Snapchat interactions and through offline discussions of those interactions, which, as we have shown, are also particularly likely to edit and embellish events, with little or no empirical evidence to drawn on.

\section{'I got, like, really creepy with it': Sexual relationships and testing boundaries}


Snaps' disappearance is Snapchat's digital trademark, yet screenshotting and saving these snaps is possible and indeed common. To explore this affordance, the participants were asked to discuss the contrast between the initial disposability presented by Snapchat and the possibility of snaps being saved, and when they thought a sent snap might be saved. Clare then told one of the most emotive and noisily-received stories of the focus groups:

CLARE: Just getting all deep on the matter, yeah, well the whole screenshotting thing is, like, how I found out my boyfriend was cheating on us. [...] Basically when we first got together, like a year ago, he had this, like girl on his Snapchat, like his top best friend and - I didn't really - I didn't know her, like, so I didn't really know anything about her, and then, she was like still in his best friends. And I was like, obviously, um, concerned as to why he was, you know, sending pictures to this girl, so, and then, um, she sent me a screenshot of a picture that he'd sent her, which was, his genitalia, [funny voice, invoking laughter from other participants] um, and sent it to me, and obviously, just like, told me, this is what's going on here.

RUTH: $\quad$ What the hell.

CLARE: Yeah. So like that's why I hate it so much, that's why I don't use it, cos, it just makes you think, like, I can remember when I got like really creepy with It, and, um he'd like come home from work, text us, and he was like, I'm just going for a shower or whatever, and then I looked on his Snapchat, and like, his, well I don't know if you can still see high scores? His score was going up and up and up and I was like, right, what's going on here? So like, obviously I knew he was speaking to people on Snapchat, like, without me knowing.

HANNAH: Yeah, that's another thing though isn't it, like, my friend, she had a boyfriend and he'd always be like 'oh I'm not Snapchatting people' and then she got a bit psycho girlfriend and was like looking at the score, and was like 'I know your score's been going up so I know you're speaking to people.'

Jurgenson's assertion that 'on Snapchat, images have no such future' (Jurgenson 2013) is a clearly a fallacy, failing to acknowledge both the screenshotting potential of such images, and 
the ways in which they shape offline lives. Snapchat's virtual reality records physical reality through pictures and activity scores. These records are ephemeral and ever-changing - Velez's brief 'bursts of affect' (Velez 2014) - but nevertheless carry huge emotional weight, and shape future relationships by providing evidence for particular activities and interactions. Clare's boyfriend's snap and activity score were used to provide proof of illicit intimate interactions, taking on lives far beyond their original intended function. Snapchat disturbs and disrupts the traditional model of psychical duration, giving particular moments in time more clarity and phantom fixity than was originally imagined.

Clare's retelling of her past is once again framed through comedy to make it bearable, and to reposition herself in a state of control and power. 'Just getting all deep on the matter' communicates that she's not really upset, while retaining a sense of gravitas. Again, the focus group operates as a tool for the malleability of Clare's duration to reshape a painful past. Speculatively, the other participants understood that Clare, at least at the time of the event, did not find it funny at all. In laughing at the appropriate moments but also providing encouragement, they become complicit in Clare's reshaping. The formality of 'genitalia' is particularly interesting; here, Clare again used a funny voice to provoke laughter, but also deliberately chose formal language, distancing herself from the intimacy of the original snap exchange. In doing so, Clare was potentially able to grasp some power over that cheating exchange, characterising it in hindsight as amusingly clinical rather than passionate - and encouraging her peers to view it in the same way.

In the focus groups, as on Snapchat, networked groups form that hold similar perspectives, and these in turn provide security, the acknowledgement that one's identity or opinion is acceptable. We have shown how group opinions can be oppressive, trapping girls in lose-lose loops of sexual agency and judgement; here, however, they are a source of strength.

It is particularly interesting to note Clare's description of her own behaviour as 'creepy', a word that invokes themes of stalkers and psychological attachment and is echoed by Hannah's phrase 'psycho girlfriend'. Such descriptions feed into another polarised discourse of appropriate femininity as performed through Snapchat, in which girls are either uptight or relaxed, and even when wronged, must respond in appropriately feminine ways.

After this episode, Clare deleted Snapchat- yet continued to check her boyfriend's activity score by looking at the app on her friends' phones.

CLARE: $\quad$...obviously everyone else still had Snapchat, so like, I'd deleted mine, and my boyfriend still had it, so like I'd text my friend and be like is high score going 
up and all this, and trying to like get, like, background information from them. But obviously like, because I'd deleted mine, it like, takes away my like, physically me knowing about it, but then, my worry and like - that it's still happening, there, but, you don't know about it. So I dunno, well it made me worry more. So, yeah I think not having Snapchat doesn't really solve anything.

As suggested earlier, Snapchat is 'too far gone now to not have it anymore'; once the app is entangled in relationships, intimacies and senses of self, it is very difficult to remove.

A power game is at play, and control can shift from one party to another through time, depending on the narratives told. Snapchat is particularly adept at encouraging this game because knowledge within the app is so ephemeral. An activity score cannot be frozen to prevent onlookers deducing that someone is Snapchatting somebody else. An illicit image cannot be protected from subsequent screenshotting. It is at once difficult to maintain secrets within Snapchat, and difficult to prove authenticity.

\section{Conclusion: Managing 'Snapchat memory': sexual exchanges, rumours and comic retellings}

The question is not whether to remember or to forget, but what to remember and to forget, and and in what context. (Grosz 2004: 118)

Snapchat is set apart from other digital 'platform vernaculars' (Gibbs et al., 2014) by is its strange temporality and ostensibly disappearing content. Explored through a Bergsonian and Deleuzian framework, we argued that what we might call 'Snapchat memory' is a murky, unreliable and changing thing and the memory image created by Snapchat has variable affects and effects for youth sexual cultures.

We found that in the absence of a digital artefact such as an image, mediated memory plays a stronger role, thus there is even greater scope for rumour and speculation. Through the mediated memories of Snapchat, girls' snaps are often read onto with sexualised content in a negative way, while boys can be seen to perform masculinity through snaps that actively imply great sexual activity and desirability. Despite the ephemerality of the snapping digital affordance, girls on Snapchat must still negotiate a fragile path between sexy and slutty. Strikingly we found girls had grown resigned to this judgement and actually viewed themselves as better able to cope with the gossip and drama surrounding discussions of sexual exchange 
on Snapchat. Thus in the case of the peer group under study we see a reinforcement of heterosexual double standards within the Snapchat user community.

Moreover, content or actions that were intended to be very transient can take on unexpected life. Far from being fleeting and disappearing as previous Snapchat research has suggested, the potential to capture snaps plays a significant role in intimate relationships. In ways similar to research on sexting when snaps do not disappear, their exchange can be used as various forms of relationship "currency" (Ringrose et al., 2013) as we saw with Clare's discovery of her boyfriend's cheating through an explicit Snap sent to another girl. The monitoring of other users' activity scores also shows us how snap data is captured and used to inform understandings of youth relationships and sexuality. These findings are of course context-specific and we have focused on understanding the implications of contemporary formations of mediated heterosexualised youth culture for the girls in our data. Studying the intersectional diversities of Snapchat use amongst differently constituted youth sexual cultures (shaped variously by ethnicity, race and religion) and finding out more about the experiences of non-heterosexual identified young people would make an important contribution to the research literature on disappearing social media.

Finally, we wish to stress that our research does not just illustrate the reproduction of heterosexism in youth digital sexual cultures. Rather, by thinking with Bergson and Deleuze and attending to the durational differences and the multiplicities generated through the Snapchat network under study, we also found evidence of resistance to dominant ways of reading the sexualised past through a logic of shame or regret, for instance (Brown and Gregg 2012). We uncovered, for example, the important role of comedy or humour in girls' mediated memory. Using humour to rethink through 'disappeared' snaps was a strategy for some of the girls to partially reform the painful episodes and moral judgements they had been subject to. As other research on women's use and development of humour online attests (Shifman and Lemish 2010), comedy perhaps enables these girls to take back some agency in an environment that places them in a sexual double standards bind. Thus, the shifting temporalities of Snapchat offer significant scope for users to continually re-mediate their memories - and specifically their intimate memories of sexualised and sexual encounters. In terms of mediated intimacy, 'Snapchat memory' offers the young people in our study an intriguing mixture of stickiness and transience, perceived permanence and elusive ephemerality. It grants them opportunities to re-shape their pasts, but never wholly and always messily. Overall, by exploring Snapchat's fast present and its ever-changing past we have demonstrated the complexity of mediation, memory and duration, providing a brief snapshot (no pun intended) into how youth are 
managing mediated sexual subjectivities and relationships in contemporary social networking cultures.

\section{List of references}

Bergson, Henri (1950: sixth impression) Time and Free Will: An Essay on the Immediate Data of Consciousness, trans. by F. L. Pogson (London: Allen \& Unwin)

Bergson, Henri (2007: resetting of 1946 edition) The Creative Mind: An Introduction to Metaphysics, trans. by Mabelle L. Andison (Mineola: Dover Publications)

boyd, danah and Jeffrey Heer (2006) 'Profiles as Conversation: Networked Identity Performance on Friendster', Proceedings of the Hawaii International Conference on System Sciences (HICSS 39)

boyd, danah and Nicole B. Ellison (2008) 'Social Network Sites: Definition, History, and Scholarship’, Journal of Computer-Mediated Communication, 13 (1), 210-230

Brown, R. \& Gregg, M. (2012) “The Pedagogy of Regret: Facebook, Binge Drinking and Young Women," Continuum 26:3 357-369.

Chambers, D. (2013) Social Media and Personal Relationships Online Intimacies and Networked Friendship, London: Palgrave.

Deleuze, Gilles (1988) Bergsonism, trans. by Hugh Tomlinson and Barbara Habberjam (Brooklyn: Zone Books)

Donath, Judith and danah boyd (2004) 'Public displays of connection', BT Technology Journal, 22 (4), 71-82

van Doorn, Niels (2010) 'The ties that bind: the networked performance of gender, sexuality and friendship on MySpace', new media \& society, 12 (4), 583-602

van Doorn, N. (2011) 'Digital Spaces, Material Traces: How Matter comes to Matter in Online Performances of Gender', Media Culture and Society, 33 (4), 531-547

van Doorn, Niels and Lisbet van Zoonen (2009) 'Theorising gender and the internet: past, present and future', in Routledge Handbook of Internet Politics, ed. by Andrew Chadwick and PhilipN. Howard (Abingdon: Routledge), pp. 261-274

Gibbs, M., Meese, J., Arnold, M., Nansen, B., \& Carter, M. (2014) '\# funeral and Instagram: Death, social media, and platform vernacular', Information, Communication \& Society, 18 (3), 255-268. doi:10.1080/1369118x.2014.987152

Goodings, L and Tucker, I M (forthcoming) 'Social media and the co-production of bodies online: Bergson, Serres and Facebook Timeline', Media, Culture \& Society

Grosz, Elizabeth (2004) The nick of time: Politics, evolution and the untimely, Durham: Duke 
University Press

Grosz, Elizabeth (2011) Becoming undone: Darwinian reflections on life, politics and art, Durham: Duke University Press

Grusin,R. (2010) Premediation: Affect and Mediality After 9/11, London: Palgrave.

Hasinoff, A. (2015). Sexting panic: Rethinking criminalization, privacy, and consent. University of Illinois Press.

Harvey, L., Ringrose, J. and Gill, R. (2013) Swagger, Ratings and Masculinity: Theorising the circulation of social and cultural value in teenage boys' digital peer networks, Sociological Research Online, 18. 4

Hillis, K. Paasonen, S and Petit, M. (2015) Networked Affect, Cambridge: MIT Press

Jurgenson, Nathan (2011) 'Digital Dualism versus Augmented Reality', The Society Pages $<$ http://thesocietypages.org/cyborgology/2011/02/24/digital-dualism-versusaugmented-reality/> Accessed 22 July 2015

Jurgenson, Nathan (2013) 'Pics and It Didn't Happen', The New Inquiry <http://thenewinquiry.com/essays/pics-and-it-didnt-happen/> Accessed 22 July 2015

Jurgenson, Nathan (2014) 'The Frame Makes The Photograph', Snapchat Blog <http://blog.snapchat.com/post/72561406329/the-frame-makes-the-photograph> Accessed 22 July 2015

Karaian, L. (2014). Policing 'Sexting': Responsibilization, respectability and sexual subjectivity in child protection/crime prevention responses to teenagers' digital sexual expression. Theoretical Criminology, 18, 282-299.

Kember, Sarah and Joanna Zylinska (2012) Life after New Media (Massachusetts: MIT Press)

Koefed, J. and Larson, M.C. (2016) A snap of intimacy: Photo-sharing practices among young people on social media, First Monday, Volume 21, Number 11 - pp. 1-9

Marwick, Alice E. and danah boyd (2014) 'Networked privacy: How teenagers negotiate context in social media, new media \& society, 16 (7), 1051-1067

Paasonen, Susanna, Ken Hillis and Michael Petit, eds. (2015) Networked Affect (Massachusetts: MIT Press)

Papacharissi, Z. (2014) Affective Publics: Sentiment, technology, politics, London: Oxford University Press

Poltash, Nicole A., (2013) 'Snapchat and Sexting: A Snapshot of Baring Your Bare Essentials', Richmond Journal of Law \& Technology 19 (4), 1-24 
Ringrose, J. (2011) Beyond Discourse? Using Deleuze and Guattari's schizoanalysis to explore affective assemblages, heterosexually striated space, and lines of flight online and at school, Educational Philosophy \& Theory, 43(6): 598-618.

Ringrose, J. and Coleman, B. (2013) Looking and desiring machines: A feminist Deleuzian mapping of affect and bodies, in B. Coleman and J. Ringrose (eds.) Deleuze and Research Methodologies, Edinburgh, EUP.

Ringrose, J., Harvey, L, Gill, R. and Livingstone, S. (2013) Teen girls, sexual double standards and 'sexting': Gendered value in digital image exchange, Feminist Theory, 14(3) $305-323$

Ringrose, J. and Harvey, L. (2017) Digital mediation, connectivity and affective materialities, in Silk, M. Andrews, D. and Thorpe, H. (2016) Routledge Handbook of Physical Cultural Studies, London: Routledge

Roesner, Franziska, Brian T. Gill and Tadayoshi Kohno (2014) 'Sex, lies, or kittens? Investigating the Use of Snapchat's Self-Destructing Messages', Proceedings of the Financial Cryptography and Data Security Conference (Christ Church, Barbados)

Senft, Theresa M. and Nancy K. Baym (2015) 'What Does the Selfie Say? Investigating a Global Phenomenon’, International Journal of Communication, 9, 1588-1606

Shifman, L and Lemish, D. (2010) 'Between feminism and fun(ny)mism: Analyzing gender in popular Internet humor', Information, Communication and Society, 13 (6), 870-891 (preprint version)

Tolman, Deborah (2012) 'Female Adolescents, Sexual Empowerment and Desire: A Missing Discourse of Gender Inequity?', Sex Roles, 66 (11-12), 746-757

Trottier, Daniel (2013) Identity Problems in the Facebook Era (Framing $21^{\text {st }}$ Century Social Issues) (London: Routledge)

Utz, Sonja, Nicole Muscanell and Cameran Khalid (2015) 'Snapchat Elicits More Jealousy than Facebook: A Comparison of Snapchat and Facebook Use', Cyberpsychology, Behavior, and Social Networking, 18 (3), 141-146

Velez, Emma (2014) 'Intimate Publics and Ephemerality, Snapchat: A Case Study', Second Shift <http://www.secondshiftblog.com/2014/09/intimate-publics-and-ephemeralitysnapchat-a-case-study/> Accessed 22 July 2015

Warfield, K. (2016) Making the cut: An agential realist examination of selfies and touch, Social Media and Society, April-June 2016: 1-10.

\section{News websites}

Gross, Doug (2013) 'Snapchat: Sexting tool, or the next Instagram?', CNN <http://edition.cnn.com/2013/01/03/tech/mobile/snapchat/index.html> Accessed 2 June 2016 
Hagan, Lucia (2016) Facebook is over. The Guardian https://www.theguardian.com/lifeandstyle/2016/dec/09/teen-guide-social-media-selfiessnapchat?CMP=fb_gu Accessed 15 December 2016

Horton, Helena (2015) 'Will Snapchat's new update stop people from sexting using the app?', Daily Telegraph <http://www.telegraph.co.uk/technology/news/11868102/WillSnapchats-new-update-stop-people-from-sexting-using-the-app.html> Accessed 2 June 2016

Jacobs, Peter (2014) 'Britain's Most Elite Boarding School Banned Snapchat To Prevent Its Students From Sexting', Business Insider <http://www.businessinsider.com/britains-mostelite-boarding-school-banned-snapchat-to-prevent-its-students-from-sexting-2014-1 ?IR=T > 